\title{
Effects of a traditional Chinese medicine formula containing the Coix seed and Lotus seed on the intestinal morphology and microbiota of local piglets
}

\author{
Zhaolong Li $i^{*}$, Zhongning Lin ${ }^{2^{*}}$, Zheng Lu ${ }^{2}$ and Zhaoyang Ying ${ }^{2}$
}

\begin{abstract}
A traditional Chinese medicine formula based on the Coix seed and Lotus seed has been used as a general treatment of malnutrition, excessive fatigue, dysfunction of the spleen and stomach, and disorders of water transport in humans in China. However, there is limited information on its effects on the gut microbiota of piglets in vivo. In this study, the mix of Coix seed and Lotus were added the diet of forty weaned piglets (local piglets), and then evaluated it's affected on the gut microbiota of piglets and on the relations within the gut bacterial community. The results indicated that this traditional Chinese medicine formula (LM) and the extract of the traditional Chinese medicine formula (LMT) downregulated $\mathrm{pH}$ of succus gastricus and raised $\mathrm{pH}$ of the ileum, and LMT obviously decreased the feed conversion ratio. Further study showed LMT and LM also significantly increased the thick and long of gastrointestinal villi. And then, 165 ribosomal DNA sequencing revealed that groups LMT and LM have higher relative abundance of the genus Lactobacillus in the colon, succus gastricus, and jejunum, which are beneficial bacteria sold as dietary supplements to aid digestion or to augment health. Meanwhile, the relative abundance levels of Prevotellaceae, Alloprevotella, and Prevotella in the colon and Clostridium in succus gastricus and jejunum were lower. These experiments highlight the usefulness of the traditional Chinese medicine formula based on the Coix seed and Lotus seed for decreasing pH in succus gastricus, for improving the structure of intestinal villi and gut microflora, and then for achieving improvements in pig production performance.
\end{abstract}

Keywords: Traditional Chinese Medicine Formula, Piglets, Gut microbiota, pH, Villus, Production performance

\section{Introduction}

Traditional Chinese medicine (TCM) is an integrative result of various bioactive compounds and shows fewer adverse effects and excellent therapeutic actions and involves a broad range of medicinal practices (Normile et al., 2003; Wang et al., 2002), which have been developed in China for thousands of years.

\footnotetext{
*Correspondence: 497377512@qq.com; 3567813@qq.com

1 Institute of Animal Husbandry and Veterinary Medicine, Fujian Academy of Agricultural Sciences, Pudang, Jin-an District, Fuzhou 350013, Fujian Province, China

${ }^{2}$ Agricultural Ecology Institute, Fujian Academy of Agricultural Sciences, Pudang, Jin-an District, Fuzhou 350013, Fujian Province, China
}

Coix seeds are mature kernels of Coix lacryma-jobi $L$. var. ma-yuen (Roman.) Stapf (Fan et al., 2017), and Lotus seeds are mature seeds of Nelumbo nucifera Gaertn (Yi et al., 2015), which are rich in starch, lipids, proteins, polysaccharides, polyphenols, phytosterols, lactams, and other bioactive ingredients and have numerous physiological and pharmacological effects fortifying the spleen; inhibiting dampness, oxidative stress, and inflammation; and regulating endocrine and immune functions (Hidaka et al., 1992; Yen et al., 2005a, b; Zhang et al., 2014; Wu et al., 2013). Accordingly, Coix seeds and Lotus seeds have pharmaceutical and food value and are widely used in China and other countries as a dietary supplement for 
daily health maintenance (Shao et al., 2009). Previously, research has demonstrated that some TCM agents can cause significant alterations of the human gut microflora and restore the balance of the gut ecosystem by inhibiting the growth of certain nonculturable bacteria and promoting proliferation of probiotics (Li et al., 2010; $\mathrm{Zu}$ et al., 2016). In recent years, some studies revealed that oligosaccharides and resistant starch of Lotus can stimulate the growth of Lactobacillus and Bifidobacterium as well as Lachnospiraceae, Ruminococcaceae, and Clostridium, respectively, and inhibit the growth of Rikenellaceae and Porphyromonadaceae in the human gut (Zhang et al., 2013, 2014; Zeng et al., 2018). Even though the Coix seed is similar to the Lotus seed and contains starch as its main component, there is limited information on the effects of the ingredients of the Coix seed on the in vivo gut microbiota (Li et al., 2019 and Liu et al., 2019), and thus, few studies have focused on the effects of a TCM formula based on the Coix seed and Lotus seed on gastrointestinal microbes of piglets.

The objective of this study was to reveal the effects of the TCM formula based on the Coix seed and Lotus seed on production performance, the structure of gastrointestinal villi, the gut microbiota of piglets, and the relations within the gut bacterial community.

\section{Materials and methods}

\section{Animals and experimental design}

Forty weaned piglets (local piglets), with weights of $7.33 \pm 0.03 \mathrm{~kg}$ (certification number: 2020000335580), were bought from Nangping Co., Ltd. and were randomly subdivided into four groups (LC, LK, LM, and LMT). The control group ( $\mathrm{LC}, \mathrm{n}=10$ ), was fed the basal diet. The piglets in the antibiotic group (LK) were provided fodder containing 1/10000 amoxicillin powder for animals (LK group, $\mathrm{n}=10$ ). The piglets in the TCM formula (LM) powder group consumed fodder containing 5/100 TCM formula powder (Table 1; LM group, $n=10$ ). The piglets in the TCM formula extract group were fed with fodder containing $1 / 100$ of the TCM formula extract (LMT group, $\mathrm{n}=10$ ). The extraction process as follows: (1) Extract: The TCM formula was added to the extraction tank, soaked in drinking water at 6 times the weight of the drug for $12 \mathrm{~h}$, and cooked twice (boiling, temperature $95-100^{\circ} \mathrm{C}$ ), water was added at 6 times of the drug weight and volume), $1.5 \mathrm{~h}$ at each time, the extract was filtered twice. (2) Concentration: The extract was concentrated at a relative density of 1.10 at $50^{\circ} \mathrm{C}$ by mixing the filtrated extract collected in step 1; (3) Drying: dextrin ( $1 \%$ by weight) was added to the concentrated liquid, which was stirred evenly and dried by spraying; the temperature in the drying tower was $90-95{ }^{\circ} \mathrm{C}$. (4) Sieve mixing: The spray-dried powder was passed through an
Table 1 Basic diet and nutrients

\begin{tabular}{ll}
\hline & $\mathbf{5 - 3 0 ~} \mathbf{~ g}$ \\
\hline Corn /\% & 50 \\
Macpi /\% & 4 \\
Soybean meal /\% & 30 \\
Fish Powder /\% & 6 \\
Premix /\% & 10 \\
Total /\% & 100 \\
Digestive Energy (DE/) (MJ. $\mathrm{Kg}^{-1}$ ) & 13.87 \\
Crude-protein (CP/\%) & 19.5 \\
Lysine acid (Lys/\%) & 1.1 \\
Calcium (Ca /\%) & 0.9 \\
Effective phosphorus (AP/\%) & 0.45 \\
\hline
\end{tabular}

80-160 mesh sieve, mixed evenly, and packaged to be used in the next step. The piglets were housed in a room with a controlled temperature $\left(23{ }^{\circ} \mathrm{C} \pm 1{ }^{\circ} \mathrm{C}\right)$, relative humidity $(60-65 \%)$, and $12 \mathrm{~h}$ light/ dark cycle (lights on at 8:00 a.m. and off at 8:00 p.m.). and all diets started on day 1 and ended on day 28. All the piglet experiments conducted in this study were in compliance with the Guidelines for the Care and Use of Laboratory Animals published by the U.S. National Institutes of Health (NIH Publication 85-23, 1996), and all the procedures were approved by the Animal Care Review Committee (Approval No. 2020000335580), Fujian Academy of Agricultural Sciences, China. The basal diet was purchased from Fujian Pajie Organism Co., Ltd. (Fujian, China). The compositions of the five diets are presented in Table 1 , and nutrition components were in accordance with GB 14924.3-2010 standards (China).

\section{Determination of the average body weight and FCR}

All piglets were weighed after overnight fasting on the morning of day 1 and on days 14 and 28 of the feeding experiment. The feed intake of each piglets was recorded daily, and then throughout the experiment to calculate the FCR.

\section{Measurement of $\mathrm{pH}$ in the gastrointestinal tract}

At the end of the $28 \mathrm{~d}$ experiment, the piglets were euthanized in accordance with the experimental animal procedures approved by the Institutional Ethics Committee/ Animal Care and Use Committee, and $\mathrm{pH}$ of succus gastricus, of the duodenum, small intestine, colon, ileum, cecum, and rectum contents were measured with a $\mathrm{pH}$ meter (PHS-5C PH, Guangzhou, China), and then statistical analysis of the recorded data was carried out. 


\section{Gastrointestinal villi}

Tissue samples $(1-2 \mathrm{~cm})$ of the stomach, duodenum, small intestine, colon, ileum, cecum, and rectum were collected from the piglets and fixed for $12 \mathrm{~h}$ in $5 \mathrm{~mL}$ of $4 \%$ paraformaldehyde. The fixed tissue was sequentially passed through $50 \%, 70 \%, 80 \%, 90 \%, 100 \%$, and $100 \%$ ethanol, for 35 to $45 \mathrm{~min}$ at each concentration and was clarified in $100 \%$ ethanol + xylene $(1: 1)$ for 30 to $40 \mathrm{~min}$. The tissue samples were then immersed in paraffin $(1: 1)$ for $30 \mathrm{~min}$. Sections of 6-12 $\mu \mathrm{m}$ thickness were prepared (average $7-8 \mu \mathrm{m}$ ), placed on slides, and stained with hematoxylin and eosin. The sections were examined, and villus length was measured under a microscope.

\section{Sample collection and extraction of genomic DNA from gastrointestinal microorganisms}

The contents of the stomach, duodenum, small intestine, colon, ileum, cecum, and rectum were collected from the piglets, and total genomic DNA from these samples was extracted by the cetyltrimethylammonium bromide (CTAB) and sodium dodecyl sulfate (SDS) method, and then DNA concentration and purity were examined on 1\% agarose gels. According to the concentration, DNA was diluted to $1 \mathrm{ng} / \mu \mathrm{L}$ with sterile water.

\section{Extraction of DNA and PCR amplification}

The samples of each above-mentioned organ of the gastrointestinal tract, approximately $100 \mathrm{mg}$, were pulverized in liquid nitrogen, resuspended in $30 \mathrm{~mL}$ of $0.1 \mathrm{~mol} / \mathrm{L}$ phosphate-buffered saline $(\mathrm{pH} 7.0)$, and stirred well. The supernatant was obtained by centrifuged three times at $12,000 \times g$ for $5 \mathrm{~min}$. Then, the supernatant was centrifuged at $12,000 \times g$ for $5 \mathrm{~min}$ at room temperature, and the bacterial pellets were collected. The pellets were resuspended in $3 \mathrm{~mL}$ of TE buffer ( $\mathrm{pH} 8.0$ ), and then the DNA was extracted with the Power Fecal DNA Isolation Kit (Mo Bio Laboratories, Inc.) according to the manufacturer's instructions. The DNA samples extracted from piglets' feces were amplified with the primer Pro $341 \mathrm{~F}$ (5'-CCTACGGGNBGCASCAG-3') and Pro 805R (5'GACTACNVGG GTATCTAATCC-3') (Nadkarni et al., 2002). All PCRs were carried out in $30 \mu \mathrm{L}$ reaction mixtures containing $15 \mu \mathrm{L}$ of the Phusion ${ }^{\circledR}$ High-Fidelity PCR Master Mix (New England Biolabs), $0.2 \mu \mathrm{M}$ of forward and reverse primers, and $\sim 10$ ng of template DNA. Thermal cycling was started with the initial denaturation at $98{ }^{\circ} \mathrm{C}$ for $1 \mathrm{~min}$; followed by 30 cycles of denaturation at $98{ }^{\circ} \mathrm{C}$ for $10 \mathrm{~s}$, annealing at $50{ }^{\circ} \mathrm{C}$ for $30 \mathrm{~s}$, and elongation at $72{ }^{\circ} \mathrm{C}$ for $60 \mathrm{~s}$; with final extension at $72{ }^{\circ} \mathrm{C}$ for $5 \mathrm{~min}$. The raw sequencing data were uploaded into the NCBI Sequence Read Archive database (SRR16895036-47).

\section{Bioinformatics analysis}

Paired-end reads were merged using FLASH (Magoc and Salzberg., 2011), which was designed to merge pairedend reads when at least some of the reads overlap (a read was generated from the opposite end of the same DNA fragment), and the splicing sequences were designated as raw tags. Quality filtering of the raw tags was performed under specific filtering conditions to obtain high-quality clean tags according to the QIIME quality control process (Bokulich et al., 2013). The tags were compared with the reference database (Gold database) via the UCHIME algorithm to detect chimeric sequences, which were later removed (Edgar et al., 2011; Haas et al., 2011). Operational taxonomic units (OTUs) were clustered at a $97 \%$ similarity cutoff in the UPARSE software (Edgar, 2013), and chimeric sequences were identified and removed using UCHIME. The taxonomic identity of each $16 \mathrm{~S}$ rRNA gene sequence was analyzed by means of RDP Classifier against the SILVA (SSU115) 16S rRNA database at a confidence threshold of 70\% (DeSantis et al., 2006). Sequences with higher than $97 \%$ similarity were assigned to the same OTU. A representative sequence for each OTU was screened for further annotation. OTU abundance data were normalized using a standard of the sequence number corresponding to the sample with the fewest sequences. Subsequent analysis of $\alpha$-diversity was performed based on these output-normalized data. $\alpha$-Diversity was analyzed as six indices: observed-species (OS), Chao1, Shannon, Simpson, ACE, and good's coverage. $\beta$-Diversity was evaluated by principal component analysis (PCA), which was conducted at phylum and OTU levels, and the hierarchical clustering tree was constructed based on Unweight Unifrac distances. All these indices for our samples were calculated in QIIME (Version 2.0) and were displayed with the R software (Version 3.2.5). The differences in a dominant bacterial community among the groups were detected via Line Discriminant Analysis (LDA) effect size (LefSe). The biomarkers used in the present study had an effect size threshold of 3.0.

\section{Statistical analysis}

To evaluate significance of the differences in the relative abundance levels of individual taxa between two groups, Metastats software (Version: 1.0) was utilized. LEfSe was used for the quantitative analysis of biomarkers within different groups. This method was designed to analyze data in which the number of species is much higher than the number of samples and to provide biological class explanations to determine statistical significance, biological consistency, and effect size estimates of the predicted biomarkers. To identify the differences in microbial communities between two groups, analysis of similarities and 
a multi-response permutation procedure were performed based on Curtis dissimilarity distance matrices.

\section{Results \\ Effects of the TCM formula on piglets' weight and feed efficiency}

Throughout the experiments, piglets of the three treatment groups-antibiotic group (LK), TCM formula group (LM), and TCM formula extract group (LMT) appeared healthier and livelier than those in the control (LC, no treatment) group. The fecal pellets were normal, and there were no deaths or diarrhea. Groups LK, LM, and LMT showed a higher average daily gain (ADG) during the $14 \mathrm{~d}$ experimental period (Table 2). Following the next $14 \mathrm{~d}$ experimental period, the highest body weight was observed in the LK group, group LM showed the lowest weight, but no differences in the ADG of piglets were observed between groups LMT and LC (Fig. 1). The feed conversion ratio (FCR) was used to describe the relation between the total weight increment and the total feed intake of piglets. After the experimental period, the FCRs of piglets in groups LC, LK, LM, and LMT were $1.722 \pm 0.013,1.498 \pm 0.018,1.643 \pm 0.021$, and $1.412 \pm 0.028$, respectively (Table 2 ). The total feed intake in the LMT group was significantly lower than in groups $\mathrm{LC}$, LK, and LM $(p<0.05)$.

\section{Effects of the TCM formula on the gastrointestinal $\mathrm{pH}$ of the piglets}

$\mathrm{pH}$ varies among different regions of the gastrointestinal tract in piglets and plays different roles in the physiological and biochemical processes. At the end of the $28 \mathrm{~d}$ experimental period, compared with the $\mathrm{LC}(\mathrm{PH}=4.86)$ and LK group $(\mathrm{PH}=4.91)$, groups $\mathrm{LM} \quad(\mathrm{PH}=3.12$, $p<0.05)$ and LMT $(\mathrm{PH}=3.35, p<0.05)$ had significantly lower $\mathrm{pH}$ in succus gastricus (Table 3 ), and higher $\mathrm{pH}$ in the ileum (LM: $\mathrm{PH}=6.57, p<0.01$; LMT: $\mathrm{PH}=6.46$, $p<0.01)$, and the $\mathrm{PH}$ of jejunum among the LMT group also has higher than other groups $(\mathrm{PH}=6.35, p<0.05)$. However, the $\mathrm{pH}$ value showed no significant differences in the caecum, colon, duodenum, and jejunum of the piglets among the three groups (Fig. 2).

\section{Evaluation of the morphological features of gastrointestinal villi}

Gastrointestinal villi and microvilli not only increase intestinal absorptive surface area but also provide a micro-environment for the growth of the gut microbiota and promote the exceptionally efficient absorption of nutrients in the lumen. In this respect, groups LM and LMT showed greater length, width, and density of villi on the gastric wall and colon wall than did groups LC and LK (Fig. 3). In addition, the villi of the cecum wall and jejunum wall in groups LM and LMT also yielded similar results. These data suggest that the Coix seed and Lotus seed may stimulate intestinal villi growth in piglets (Fig. 4).

\section{Composition differences of gut microbiota among four groups}

Sequencing analysis indicated that species richness of gut microbiota on LK group was lowest than other groups (Chao1 on colon: LK, 25,104.533 \pm 109.407 , LC, 28,094.745 $\pm 208.352 ; \quad$ LM, 27,288.283 \pm 213.735 ; and LMET, 26,326.204 \pm 276.666$)$ and other indexes showed similar results, such as Shannon (Colon of LK, $12.411 \pm 1.375$; Caecum of LK, 12.890 \pm 1.627 ; Succus gastricus of LK, $10.967 \pm 1.097$; Jejunum of LK $9.752 \pm 1.075)$, but the gastro-intestinal species richness of LC 、 LM and LMT groups shown higher (Table 4). Ace indexes also similar results. We used unweighted UniFrac distances to separate the LM 、 LMT and LK group. The PCoA explained 9.53 and $61.24 \%$ variation among four groups piglets for unweighted UniFrac distances,

Table 2 Comparison of the growth performance of the local piglets

\begin{tabular}{|c|c|c|c|c|}
\hline & LMT & LM & LK & Control \\
\hline Total food intake (kg) & 118.65 & 118.6 & 142.85 & 139.9 \\
\hline Average daily food intake (ADFI) (kg/per head) & 0.42 & 0.42 & 0.51 & 0.50 \\
\hline Total additive addition amount (kg) & 5.93 & 5.93 & 7.14 & 0 \\
\hline Actual additive ratio (\%) & 5 & 5 & 0.05 & 0 \\
\hline Initial weight (kg) & $7.36 \pm 0.58$ & $7.36 \pm 0.55$ & $7.37 \pm 0.78$ & $7.38 \pm 0.28$ \\
\hline Last weight (kg) & $15.76 \pm 3.00$ & $14.58 \pm 2.34$ & $16.9 \pm 2.05$ & $15.5 \pm 2.54$ \\
\hline Average weight gain $(\mathrm{kg})$ & $8.4 \pm 1.24$ & $7.22 \pm 1.29$ & $9.54 \pm 2.84$ & $8.13 \pm 1.48$ \\
\hline Average daily gain (ADG) (kg/per head) & $0.30 \pm 0.15$ & $0.26 \pm 0.34$ & $0.34 \pm 0.17$ & $0.29 \pm 0.10$ \\
\hline Feed/Weight gain ratio (F/G) & 1.412 & 1.643 & 1.498 & 1.722 \\
\hline F/G vs. control group comparison & $\downarrow 0.31(18 \%)$ & $\downarrow 0.079(4.6 \%)$ & $\downarrow 0.224(13.00 \%)$ & \\
\hline
\end{tabular}




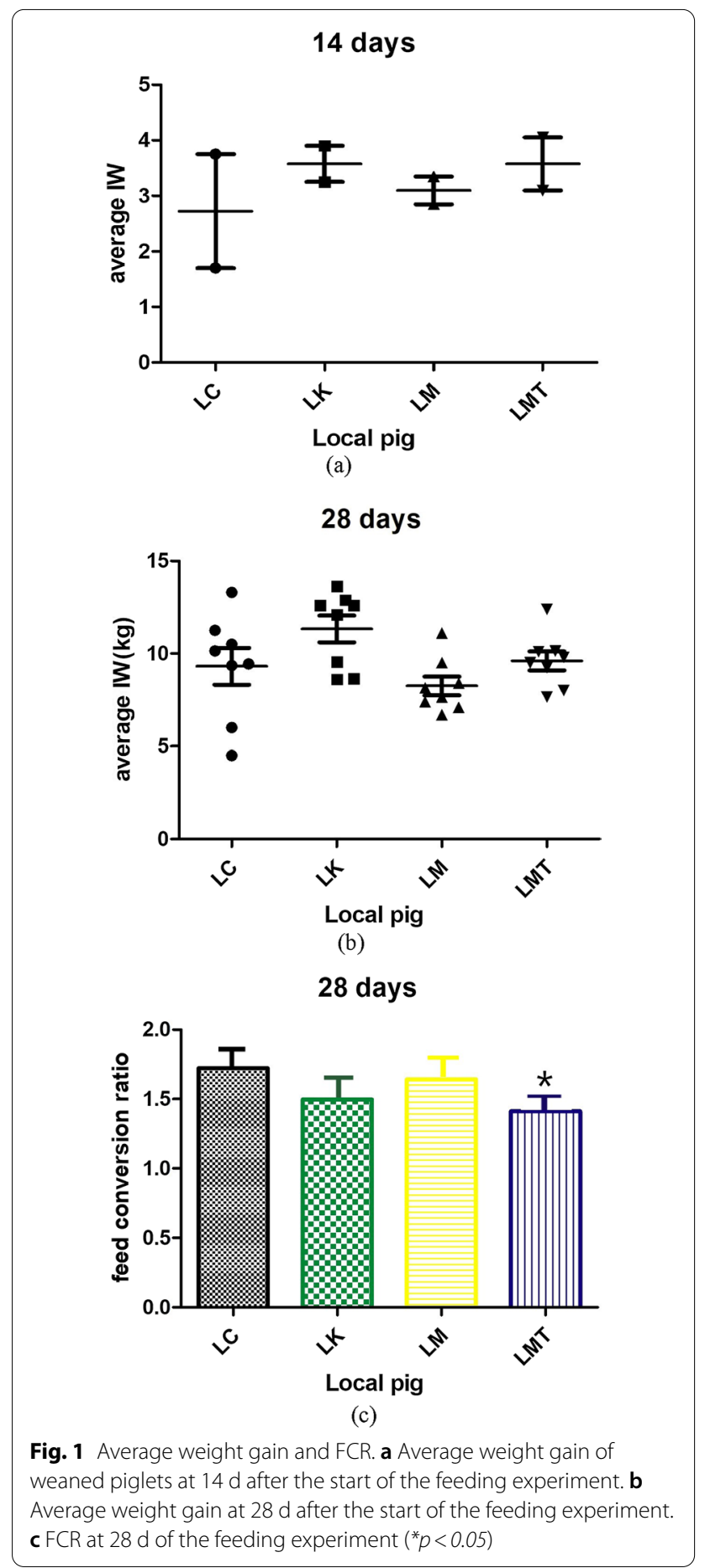

respectively (Fig. 5). The inter-individual beta diversity varied significantly $(p<0.001)$ in all the four group piglets. Among TCM groups (LM and LMT group) shown higher beta diversity index than LK group, which indicates high level of variety in the microbial composition of LM and LMT group piglets (Fig. 5).
Table $3 \mathrm{pH}$ of the gastrointestinal content of weaned piglets on day 28

\begin{tabular}{lllll}
\hline Site & LMT & LM & LK & LC \\
\hline Caecum & $6.26 \pm 2.23$ & $5.59 \pm 0.98$ & $5.64 \pm 0.23$ & $5.48 \pm 0.23$ \\
Colon & $5.86 \pm 2.03$ & $5.78 \pm 2.79$ & $5.9 \pm 0.29$ & $5.51 \pm 1.36$ \\
Duodenal & $6.14 \pm 1.64$ & $5.82 \pm 2.22$ & $5.74 \pm 1.51$ & $5.63 \pm 1.56$ \\
lleum & $6.50 \pm 1.15$ & $6.66 \pm 1.38$ & $5.84 \pm 1.16$ & $5.59 \pm 1.15$ \\
Succus gastricus & $3.15 \pm 1.84$ & $3.36 \pm 1.56$ & $5.04 \pm 1.49$ & $5.01 \pm 1.50$ \\
Jejunum & $6.38 \pm 1.89$ & $5.87 \pm 1.76$ & $5.75 \pm 1.62$ & $5.47 \pm 1.35$ \\
\hline
\end{tabular}

\section{Comparison of the predominant bacterial community structure in the colon among the groups}

To reveal the effects of the TCM formula on the colon microbiota of piglets, 16S rRNA sequencing was carried out on the Illumina technology platform. From day 14 to day 28, the fold increases in the relative abundance of Prevotellaceae NK3B31(0.727, $p<0.001)$, Alloprevotella $(0.946, p<0.01)$, and Prevotella-9 $(0.246, p<0.001)$ in the LM group were significantly lower than those in the other groups (Fig. 6), especially the fold increase of Prevotella_9 abundance was the lowest. In addition, compared with the LK group (0.133), groups LM $(0.946, p<0.001)$, LMT $(0.984, p<0.001)$, and LC $(0.997, p<0.001)$ showed greater fold increases in the relative abundance of Lactobacillus at 28 and $14 \mathrm{~d}$, and Lactobacillus growth in the LK group was the slowest.

\section{Comparison of the predominant bacterial community structure in the caecum among the groups}

To uncover the effects of the Coix seed and Lotus seed on the bacterial community of the cecum in piglets, we collected cecum content for analysis on the 14th and 28th $\mathrm{d}$ of the experiment, and compared these data (Fig. 7). Groups LM and LMT featured lower abundance of Clostridium and Prevotellaceae NK3B31 (LM:1.034, $p<0.001$; LMT:1.181, $p<0.001)$. The LK group showed a significant increase in Prevotellaceae NK3B31 abundance $(2.982, p<0.001)$ in the cecum of piglets. Clostridium in the LC group $(3.327, p<0.001)$ was up to fourfold more abundant at $28 \mathrm{~d}$ than at $14 \mathrm{~d}$, but groups LK (0.795), LM (0.698), and LMT (0.895) did not show obvious up-regulation.

\section{Effects of the formula based on the Coix seed and Lotus seed on the bacteria in the stomach}

Microorganisms in the stomach and jejunum secrete various enzymes to promote the absorption of nutrients, and thus, the difference in the microbial community between the stomach and jejunum plays an important role in the health of piglets. Microbiota analysis of piglets' stomach indicated that in the LMT group $(0.655, p<0.001)$, the 


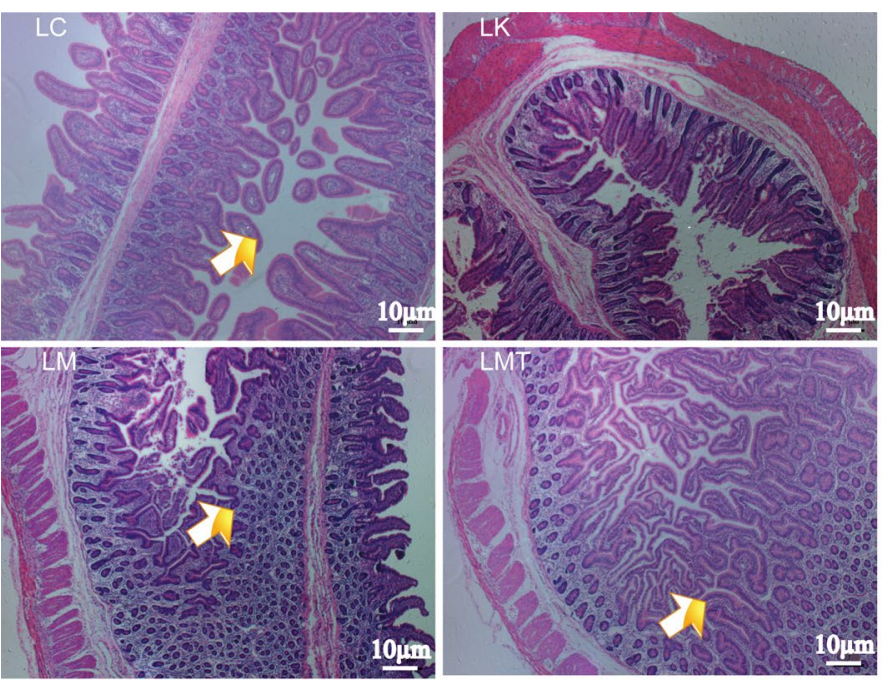

(a)

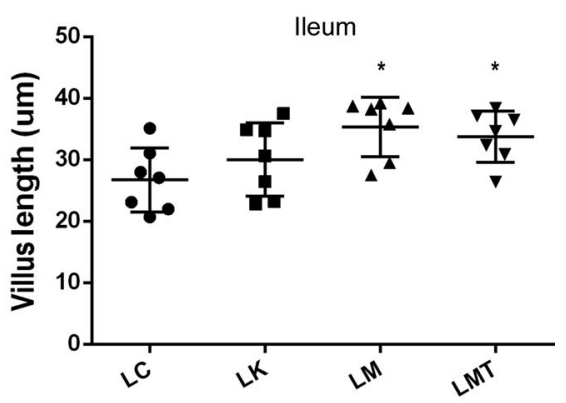

(b)
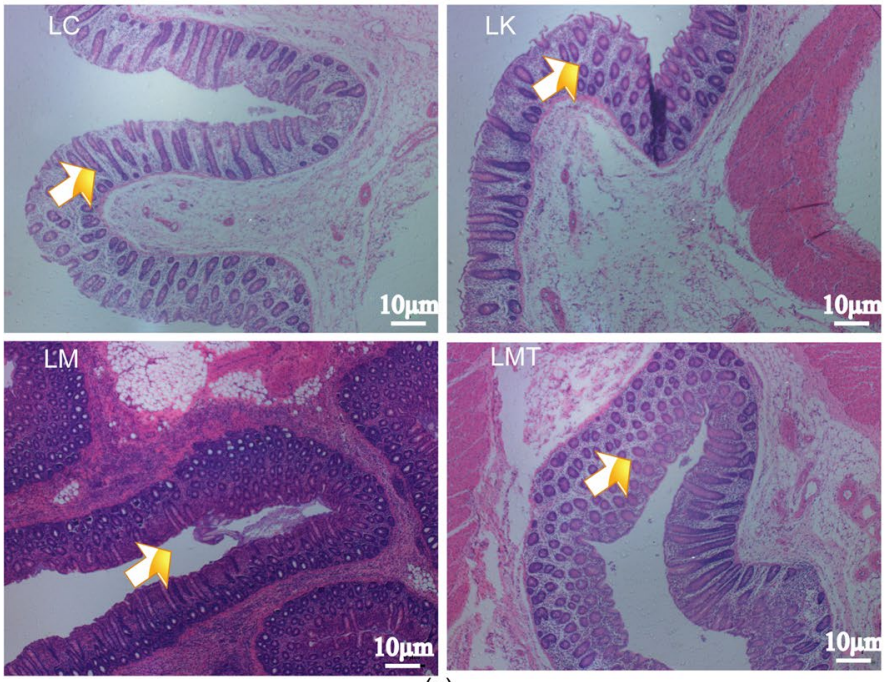

(c)

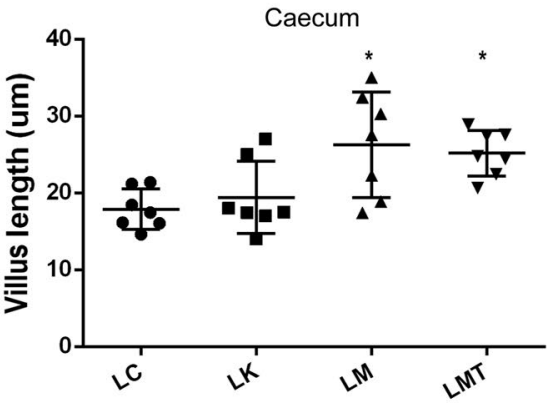

(d)

Fig. 2 Variation in $\mathrm{pH}$ of the gastrointestinal content of weaned piglets on day 28. a pH levels of succus gastricus, $\mathbf{b} \mathrm{pH}$ of rectal content, $\mathbf{c} \mathrm{pH}$ of cecal content, $\mathbf{d} \mathrm{pH}$ of ileal content, $\mathbf{e} \mathrm{pH}$ of duodenal content, $\mathbf{f} \mathrm{pH}$ of jejunal content, and $\mathbf{g} \mathrm{pH}$ of colonic content $\left({ }^{*} p<0.05\right)$

abundance of Lactobacillus enormously increased than other groups, thus the LK group $(0.1430, p<0.05)$ was the lowest. The fold increase in Clostridium_sensus_stricto_1 relative abundance was the highest in the LK group (5.742, $p<0.001$ ) (Fig. 8), and other groups not clearly differences each other. In this study, the abundance of
Lactobacillus in the LMET group $(3.493, p<0.001)$ enormously increased in the jejunum. Groups LC, LK, and LM clearly showed the down-regulation of Lactobacillus in the jejunum. And then, the fold increase of Clostridium_sensus_stricto_1 relative abundance was the highest in the LC group with 4.810 (Fig. 9), and the abundance in 

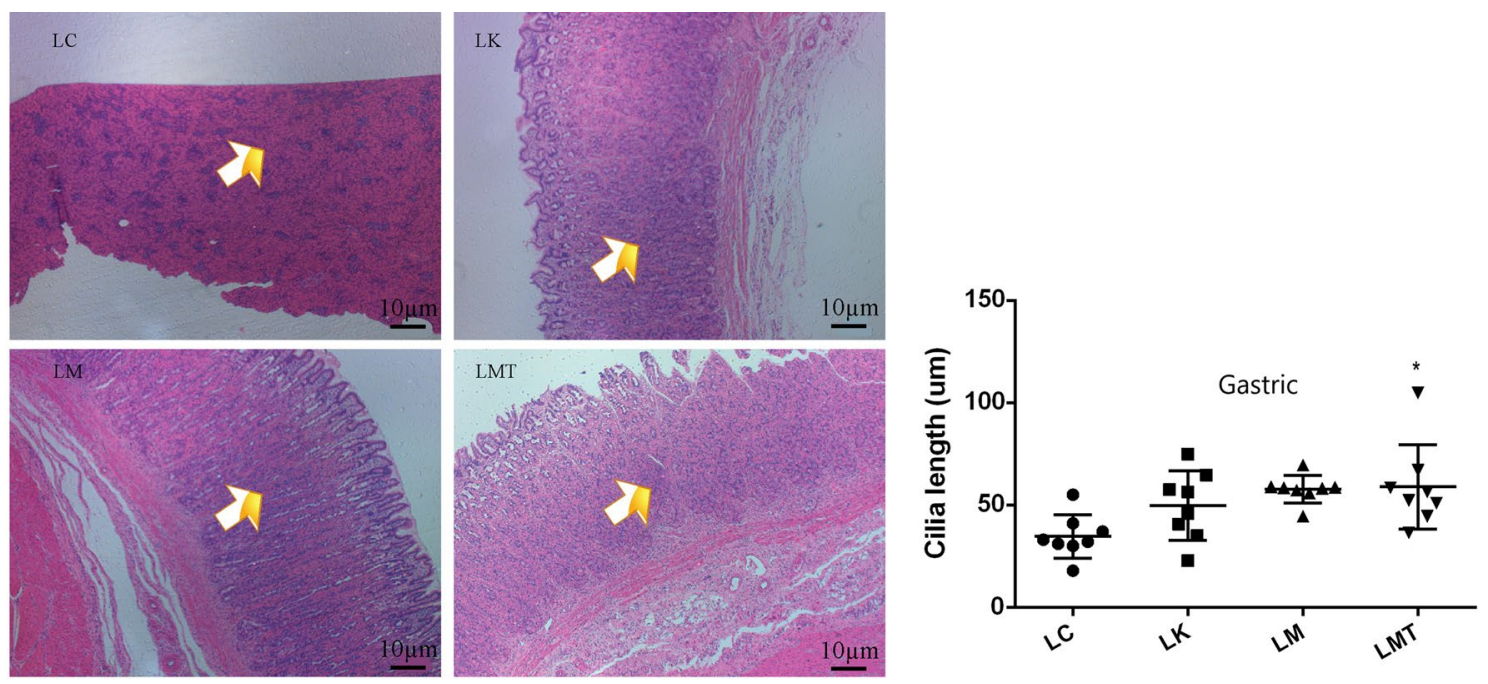

(a)

(b)
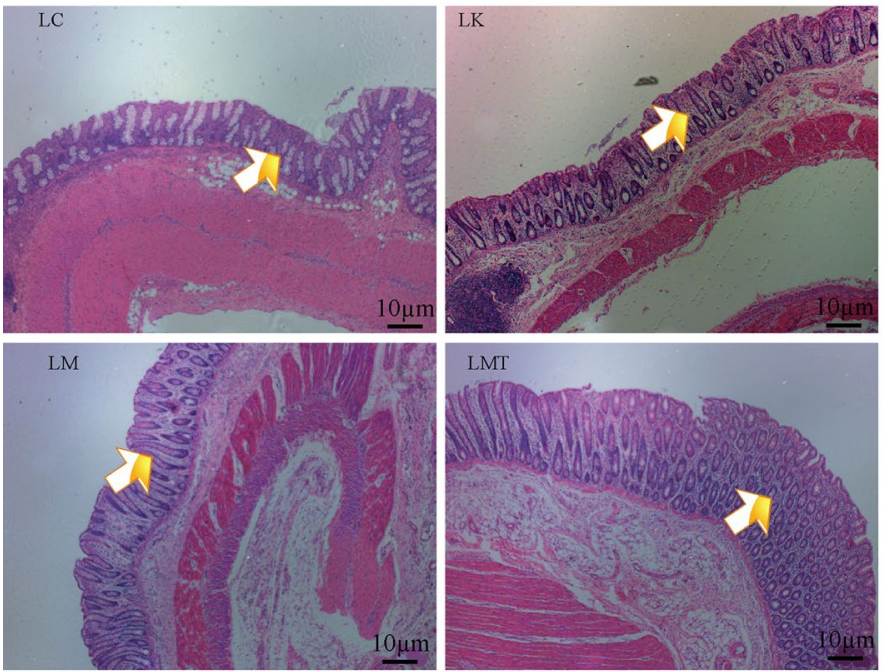

(c)

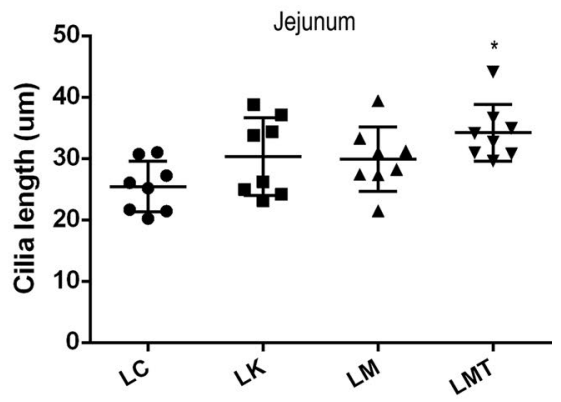

(d)

Fig. 3 Morphological changes in gastrointestinal villi of the weaned piglets. a Morphological changes of gastric villi in the weaned piglets. $\mathbf{b}$ The length of gastric villi. $\mathbf{c}$ Morphological changes in the jejunum of the weaned piglets. $\mathbf{d}$ The lengths of jejunal villi in different groups $\left({ }^{*} p<0.05\right.$; $\left.{ }^{* *} p<0.02 ;{ }^{* * *} p<0.01\right)$

$\mathrm{LK}(2.600, p<0.001)$ and LM $(2.811, p<0.001)$ groups significantly decreased between days 14 and 28 (Fig. 9), but the LMT group has one sample which has higher record with 15.442, if the data is excluded, its average was 3.042 be closed the LK and LC groups.

\section{Discussion}

TCM formulations as dietary supplements have positive effects on the growth performance of piglets: previous reports have demonstrated that dietary supplementation with the TCM herbs can increase average daily feed 


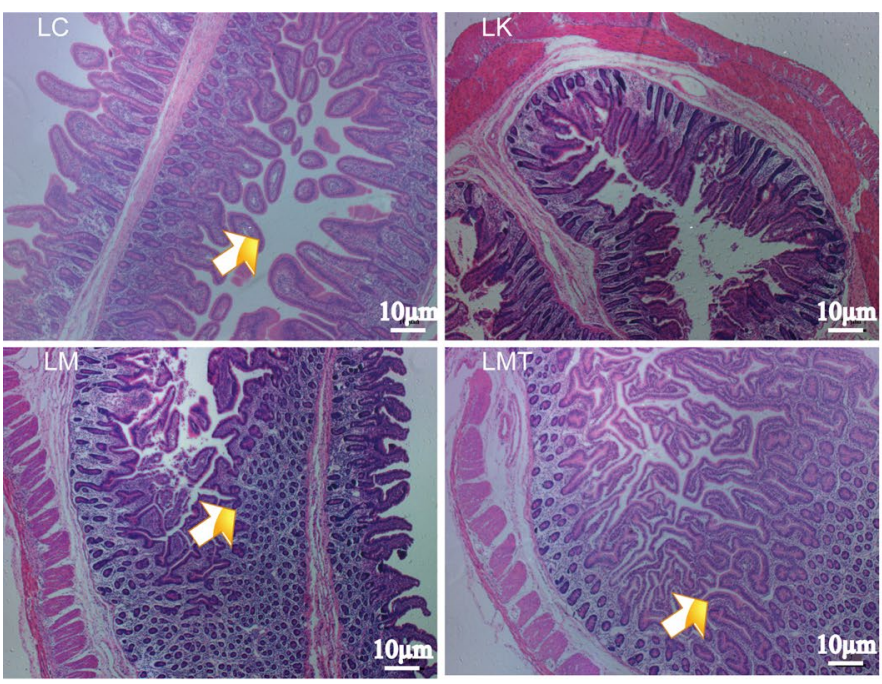

(a)

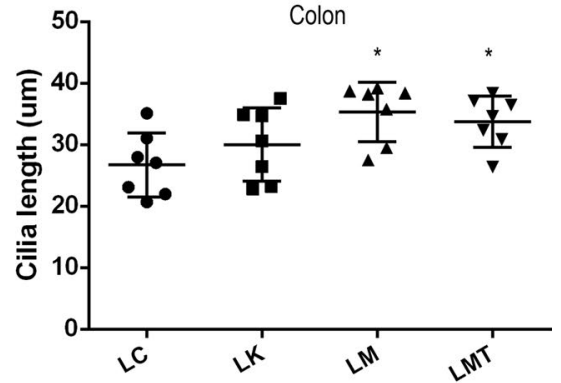

(b)
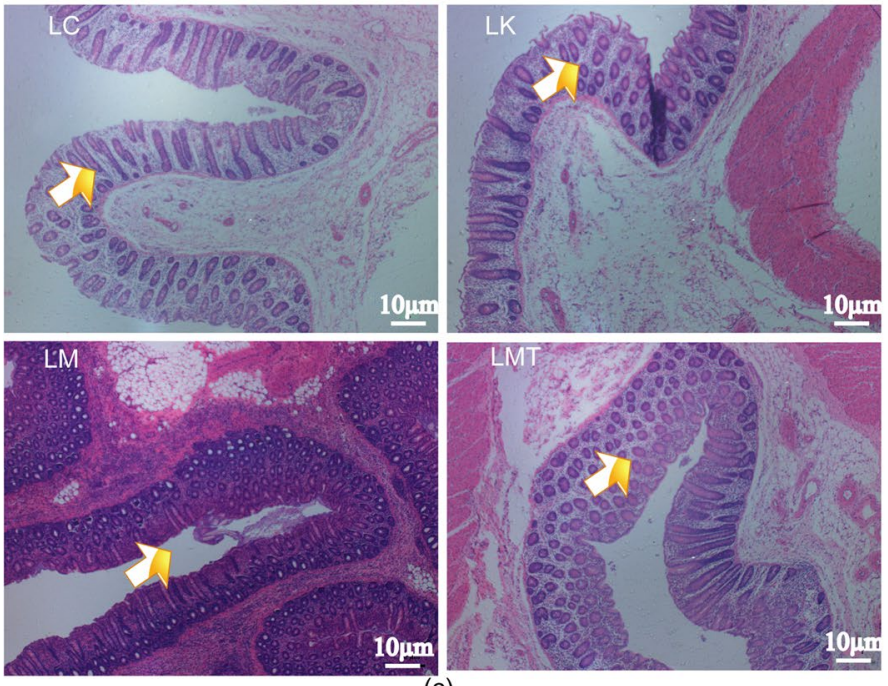

(c)

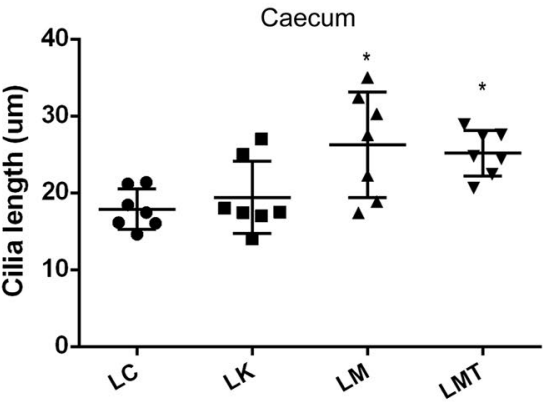

(d)

Fig. 4 Morphological changes in the cecal and colonic villi of weaned piglets. a Morphological changes in the cecum of the weaned piglets. $\mathbf{b}$ Length of colon villi. c Morphological changes in the cecal of weaned piglets. $\mathbf{d}$ Length of colonic villi in different groups $\left({ }^{*} p<0.05\right.$; $\left.{ }^{* * *} p<0.01\right)$

intake and ADG, improve the gain/feed ratio, and reduce the incidence of diarrhea in weaned piglets (Chen et al., 2007; Lin et al., 2013; Xu et al., 2012; Zhao et al., 2015; Jian et al., 2007). Here, our results suggest that the LMT group manifested a significant increase in ADG, and the FCR tended to diminish in these piglets as compared to that in the LC group, in agreement with previous research (Lai et al., 2015; Gui et al., 2007). This result can be explained as follows: the TCM formula extract is rich in starch, polysaccharides, and protein, which promoted the growth of piglets. Another important factor is that the TCM formula extract reverses the dysfunction of 
Table 4 Alpha diversity indices

\begin{tabular}{lrrr}
\hline Group & \multicolumn{1}{l}{ Shannon } & \multicolumn{1}{l}{ Chao 1} & \multicolumn{1}{l}{ Ace } \\
\hline LCE_JC & $13.022 \pm 1.253$ & $28,094.745 \pm 208.352$ & $29,113.150 \pm 256.423$ \\
LKE_JC & $12.411 \pm 1.375$ & $25,104.533 \pm 109.407$ & $26,086.854 \pm 258.355$ \\
LME_JC & $12.655 \pm 2.112$ & $27,288.283 \pm 213.735$ & $28,431.517 \pm 357.526$ \\
LMTE_JC & $12.636 \pm 1.143$ & $26,326.204 \pm 276.666$ & $26,392.808 \pm 278.177$ \\
LCE_MC & $12.569 \pm 1.009$ & $26,411.583 \pm 354.109$ & $27,580.895 \pm 286.396$ \\
LKE_MC & $12.890 \pm 1.627$ & $27,295.407 \pm 321.423$ & $28,054.284 \pm 439.329$ \\
LME_MC & $12.834 \pm 0.988$ & $27,811.435 \pm 432.767$ & $28,716.256 \pm 397.491$ \\
LMTE_MC & $12.241 \pm 1.172$ & $24,683.871 \pm 307.611$ & $25,777.705 \pm 303.776$ \\
LCE_WY & $11.099 \pm 1.053$ & $16,422.965 \pm 199.865$ & $17,168.775 \pm 245.980$ \\
LKE_WY & $10.967 \pm 1.097$ & $17,937.138 \pm 303.123$ & $18,781.656 \pm 277.313$ \\
LME_WY & $11.911 \pm 1.564$ & $20,529.737 \pm 206.639$ & $21,043.125 \pm 331.694$ \\
LMTE_WY & $11.674 \pm 2.053$ & $20,441.081 \pm 193.708$ & $21,566.082 \pm 425.101$ \\
LCE_XC & $10.784 \pm 1.122$ & $13,793.828 \pm 177.067$ & $14,423.189 \pm 222.039$ \\
LKE_XC & $9.752 \pm 1.075$ & $9718.633 \pm 252.278$ & $9951.928 \pm 355.228$ \\
LME_XC & $10.176 \pm 1.636$ & $11,670.409 \pm 351.185$ & $12,139.562 \pm 211.371$ \\
LMTE_XC & $10.387 \pm 1.405$ & $13,518.902 \pm 407.534$ & $14,699.148 \pm 197.012$ \\
\hline
\end{tabular}

the spleen and stomach and disorders of water transport and corrects malnutrition. Nevertheless, in this study, we found that the FCR values of the LK group also followed a clearly decreasing trend approaching that of the LMT group. Therefore, appropriate addition of antibiotics can correct an intestinal imbalance and thus promote the growth performance of piglets (Nemechek et al., 2013).

$\mathrm{pH}$ levels in the gastrointestinal tract of piglets play an important role in the maintenance of normal physiological function of the digestive system and regulate the acid-base balance and electrolyte balance in the inner circumferential environment (Schnabel et al., 1982; Clark et al., 2010; Straw et al., 1991). Low pH in the stomach can accelerate the conversion of pepsinogen to pepsin and expansion and denaturation of feed protein; these processes are beneficial for the digestion of feed protein (Burnell et al., 1988). In addition, low pH in the stomach can slow down the gastric emptying rate, delay the residence time of feed in the stomach, and promote the digestion and absorption of nutrients (Clark et al., 1993). The present study also revealed that the $\mathrm{pH}$ values of the stomach were lower in groups LMT and LM than in groups LK and LC; these data are consistent with the growth performance mentioned above (Suiryanrayna et al., 2015).
It is known that an enormous number of exogenous and internal factors mediate the regulation of $\mathrm{pH}$ in the gastrointestinal tract. Among them, the diet is an important exogenous factor. According to the latest reports, stomach acidity is mainly affected by the buffering capacity of solid food and water (Meler A et al., 2012; Kong et al., 2009). In recent years, more and more studies suggest that herbs and extracts help to regulate $\mathrm{pH}$ of the gastrointestinal tract (Xu et al., 2009). In the present study, groups LMT and LM showed lower $\mathrm{pH}$ in the stomach of piglets, in line with previous research $(\mathrm{Xu}$ et al., 2009). The TCM formula and TCM formula extract acted as nutritional supplements that regulate gastrointestinal $\mathrm{pH}$. Nevertheless, $\mathrm{pH}$ of the ileum in groups $\mathrm{LM}$ and LMT ended up at 6.8, which was clearly higher than $\mathrm{pH}$ in the LK and LC groups.

The gastrointestinal villi of piglets not only act as a tissue for food digestion and absorption but also play an important part in the fight against pathogenic bacteria and toxic substances present in the intestinal lumen (Yang et al., 2013). Therefore, ensuring the normal development and growth of intestinal villi is the key to improving the growth performance of piglets. Previous research suggests that weaning is substantial stress for piglets and is accompanied by a reduction in villus height and worsening of villus morphology, and the small intestine continues to deteriorate until the fifth day after weaning (Miller et al., 1986). To reduce the effects of stress on piglets, many authors have suggested that TCM supplements can increase villus height (reduce villous atrophy) and crypt depth (reduce crypt hyperplasia), and a lot of studies have indicated that some TCM formulations can promote the development and growth of intestinal villi (Yang et al., 2013). In this study, groups LM and LMT showed a subsequent increase in the villus height in the stomach and cecum the whole experiment; this finding is also consistent with the growth performance mentioned above (Shi et al., 2015).

According to the influence of the intestinal microflora on host health, it can be subdivided into probiotics, harmful bacteria, and opportunistic bacteria (Clemente et al., 2012). While probiotics such as Bacteroidetes and Lactobacillus dominate in the host intestine, the host is in a relatively healthy state. A large number of studies have shown that TCM can significantly alleviate intestinal microflora disorders, promote the growth of beneficial 


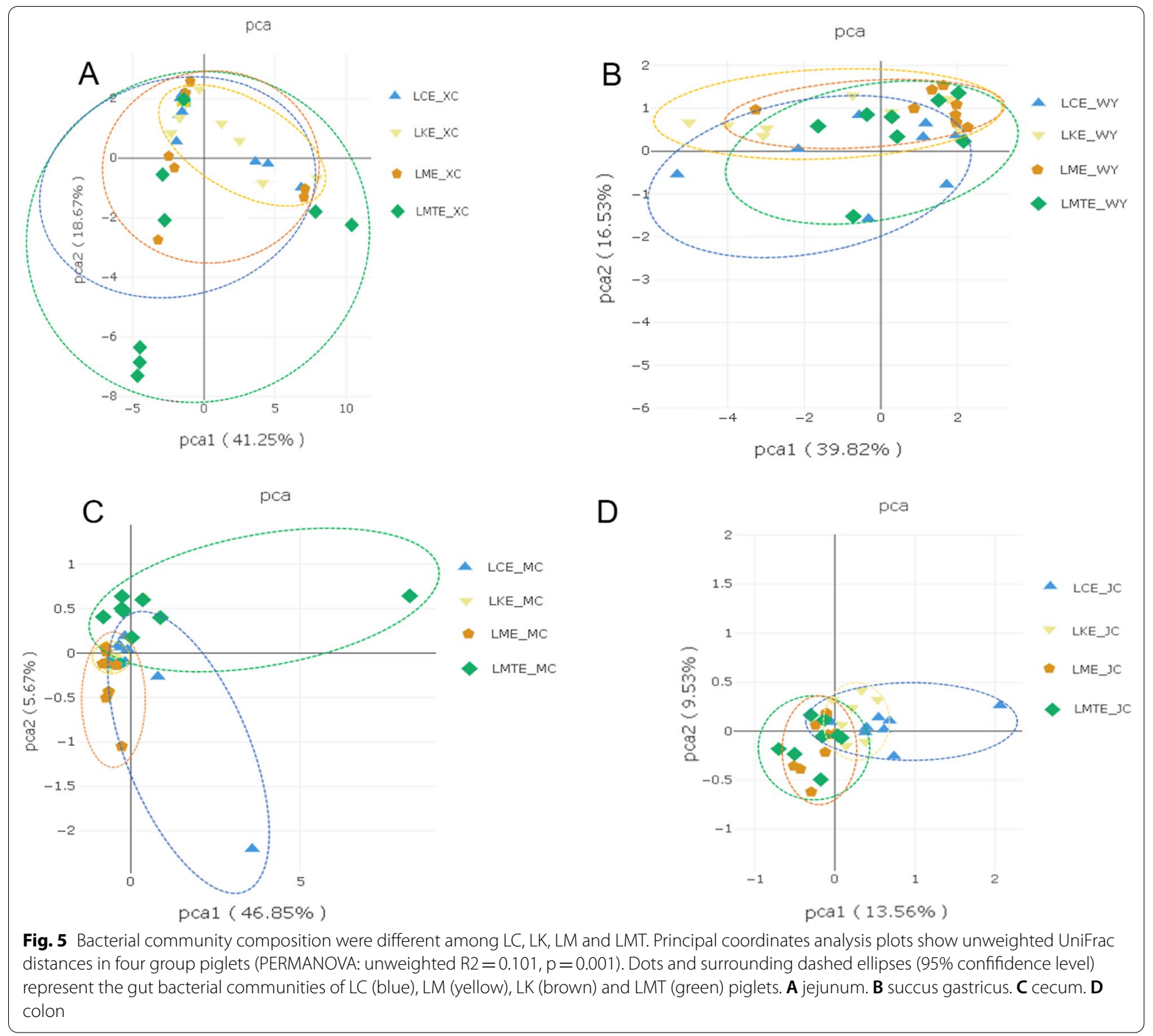

bacteria, inhibit the excessive propagation of harmful bacteria, and balance the abundance of these probiotics and pathogenic bacteria, thus maintaining a healthy environment in the intestinal tract (Li et al., 2010; He et al., 2007). For example, polysaccharides, an active ingredient of TCM formulations, can improve the intestinal environment, contribute to the growth of probiotics, and may play the role of a prebiotic, which is beneficial to the growth of probiotics (Wang et al., 2015). These prebiotics promote the growth of probiotics by changing the growth environment of the intestinal flora or by serving as a substrate, and some organic acids in TCM formulations 


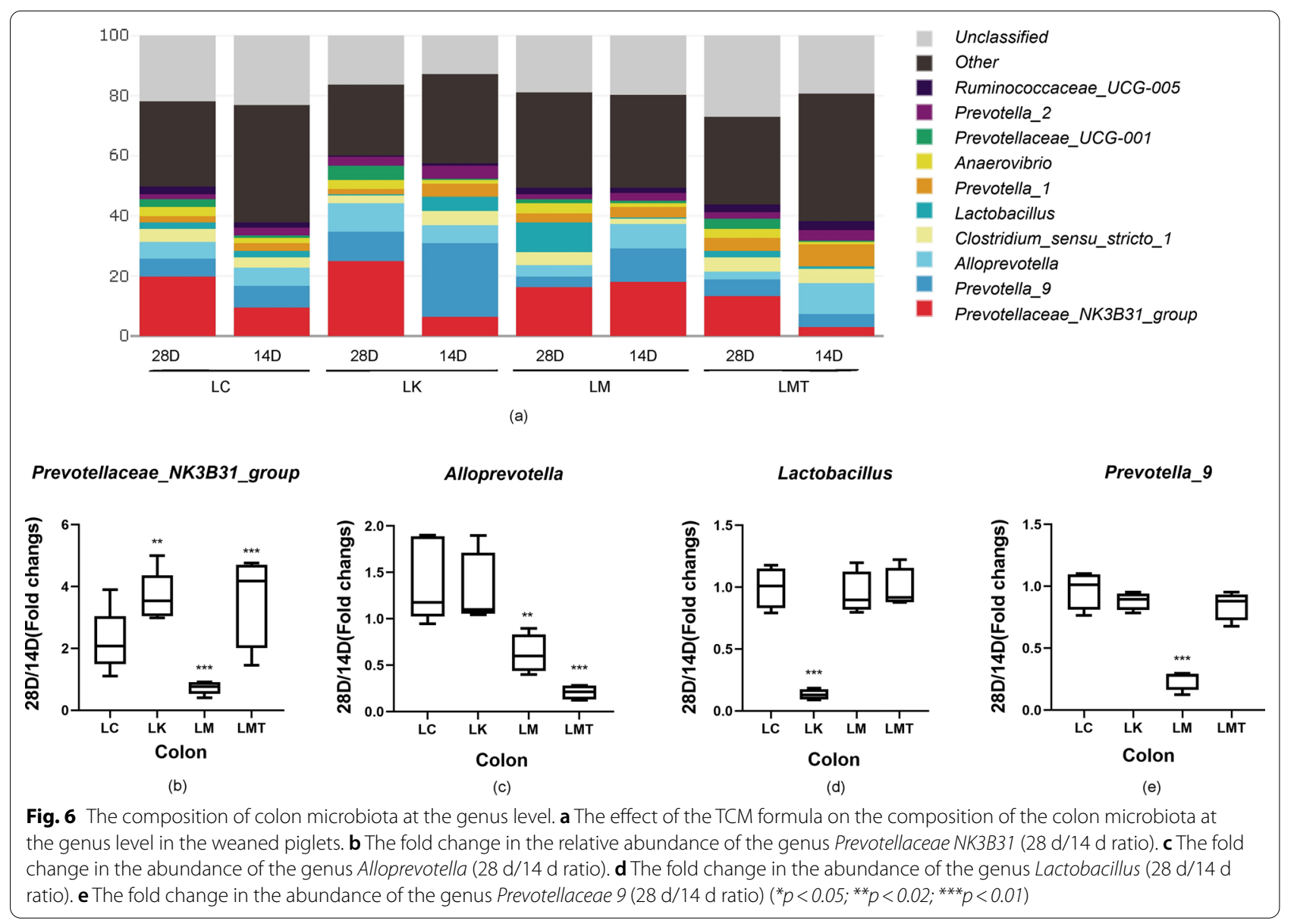

act as $\mathrm{pH}$ buffers to maintain the stability of $\mathrm{pH}$ in the intestinal tract to provide a suitable living environment for probiotic proliferation (Modesto et al., 2009). In particular, herbal supplements such as ginseng, Ganoderma lucidum, Lotus seed, Coix seed, and Codonopsis pilosula can promote the growth of probiotics to a certain extent (Koo et al., 1994; Zeng et al., 2018; Liu et al., 2019). Their ingredients can accelerate the growth of probiotics (e.g., Bifidobacterium, Lactobacillus, and Streptococcus thermophilus) after entering the intestine (Li et al., 2010). In the present study, the TCM formulae used in groups LM and LMT are based on the Lotus seed and Coix seed, which are rich in prebiotics such as amino acids, polysaccharides, and starch(Xi et al., 2019; Xu et al., 2018). These formulations also significantly promote the proliferation of Lactobacillus in the colon, jejunum, and stomach.

In addition, TCM herbs can inhibit the growth of pathogenic microorganisms, especially the harmful bacteria 


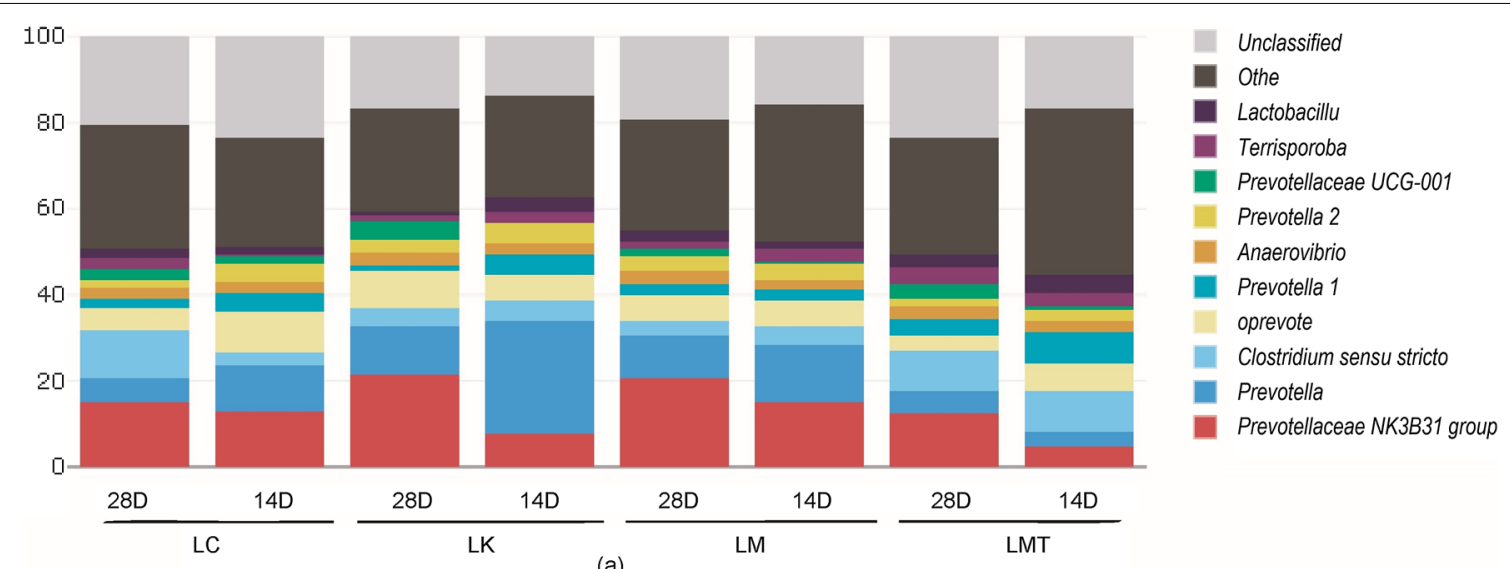

Prevotellaceae_NK3B31_group

Clostridium_sensu_stricto_1

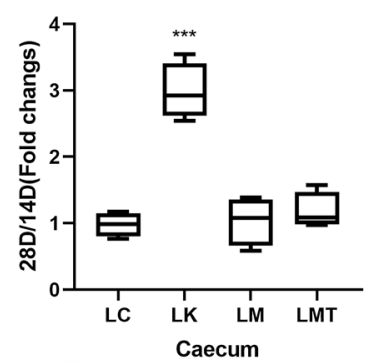

(b)

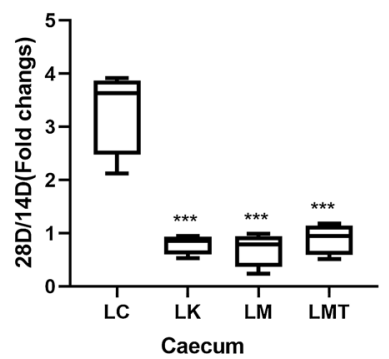

(c)

Fig. 7 Composition of the cecum microbiota at the genus level. a1 The effect of the TCM formula on the composition of the cecum microbiota at the genus level in the weaned piglets. b The fold change in the relative abundance of the genus Prevotellaceae NK3B31 (28 d/14 d ratio). c The fold change in the abundance of the genus Clostridium sensu stricto 1 (28 d/14 d ratio) ${ }^{*} p<0.05$; ${ }^{* *} p<0.02 ;{ }^{* * *} p<0.01$, )

in the intestinal tract (Wang et al., 2010; Xuan et al., 2014; Chandrakar et al., 2015). This study also proved that treatments LM and LMT significantly inhibited the growth of Prevotella, Alloprevotella, and Clostridium. On the contrary, in the LK group and LC group, there was an obvious increase in the relative abundance of microorganisms. Particularly, the LK group showed higher relative abundance of stomach Clostridium. Clostridium spp. belongs to firmicutes, which is a large class of normal facultative anaerobic bacteria in intestine. Clostridium mainly was consists of two types: harmful and beneficial. Dozens of Clostridium strains have been reported in previous studies. Some of them were used to prevent, diagnose and treat relative human diseases, and others were demonstrated to be related to diseases including antibiotic-associated diarrhea, pseudomenbraneouscolitis and 


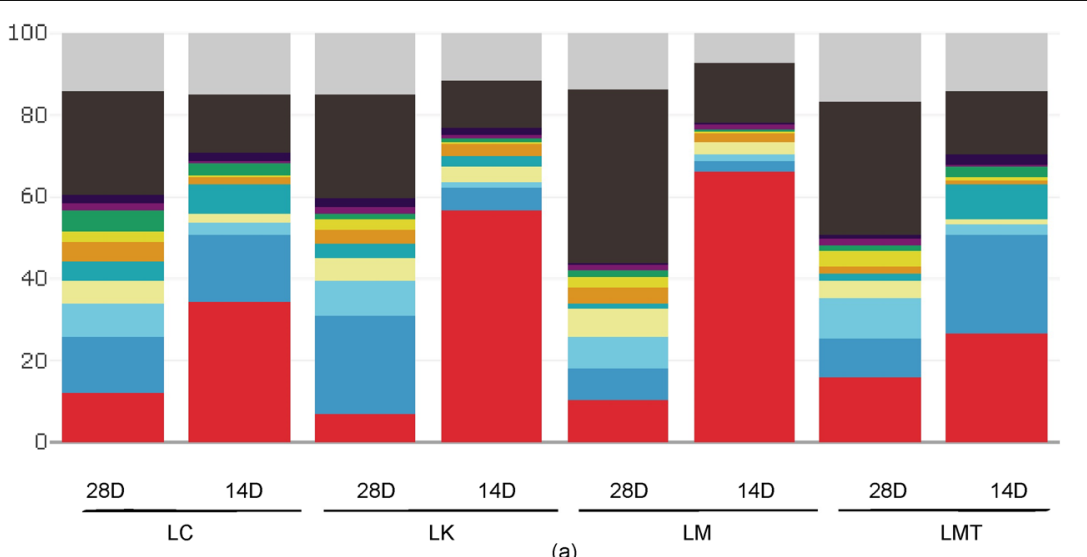

II Unclassified

- Other

- Oryza_meyeriana

- Alloprevotella

Streptococcus

Terrisporobacter

Prevotella_9

Bromus_tectorum

Prevotellaceae_NK3B31_group

I Clostridium_sensu_stricto_1

- Phaseolus_acutifolius

Lactobacillus

(a)

Clostridium_sensu_stricto_1

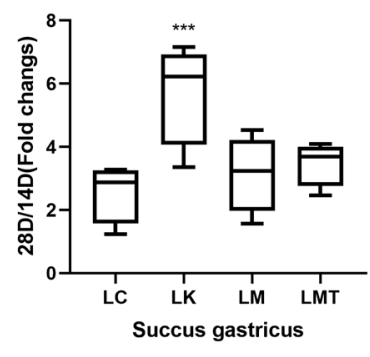

(b)
Lactobacillus

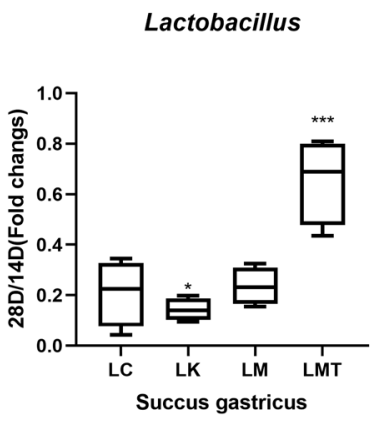

(c)

Fig. 8 The composition of the succus gastricus microbiota at the genus level. a The effect of the TCM formula on the composition of the succus gastricus microbiota at the genus level in the weaned piglets. b The fold change in the relative abundance of the genus Clostridium sensu stricto 1 (28 d/14 d ratio). c The fold change in the abundance of the genus Lactobacillus (28 d/14 d ratio) $\left({ }^{*} p<0.05 ;{ }^{* *} p<0.02 ;{ }^{* * *} p<0.01\right)$

so on. In this study, whether the genus Clostridium in LK group is a pathogenic microbe need to be demonstrated in the further study.

The TCM formula based on the Coix seed and Lotus seed not only reduces gastrointestinal $\mathrm{pH}$ and increases the villus height but also promotes the growth of probiotics. Additionally, it inhibits the proliferation of pathogenic microorganisms and thus improves production performance of piglets. The proposed nutritional supplement seems to be an appropriate substitute for antibiotics in piglets feed. 


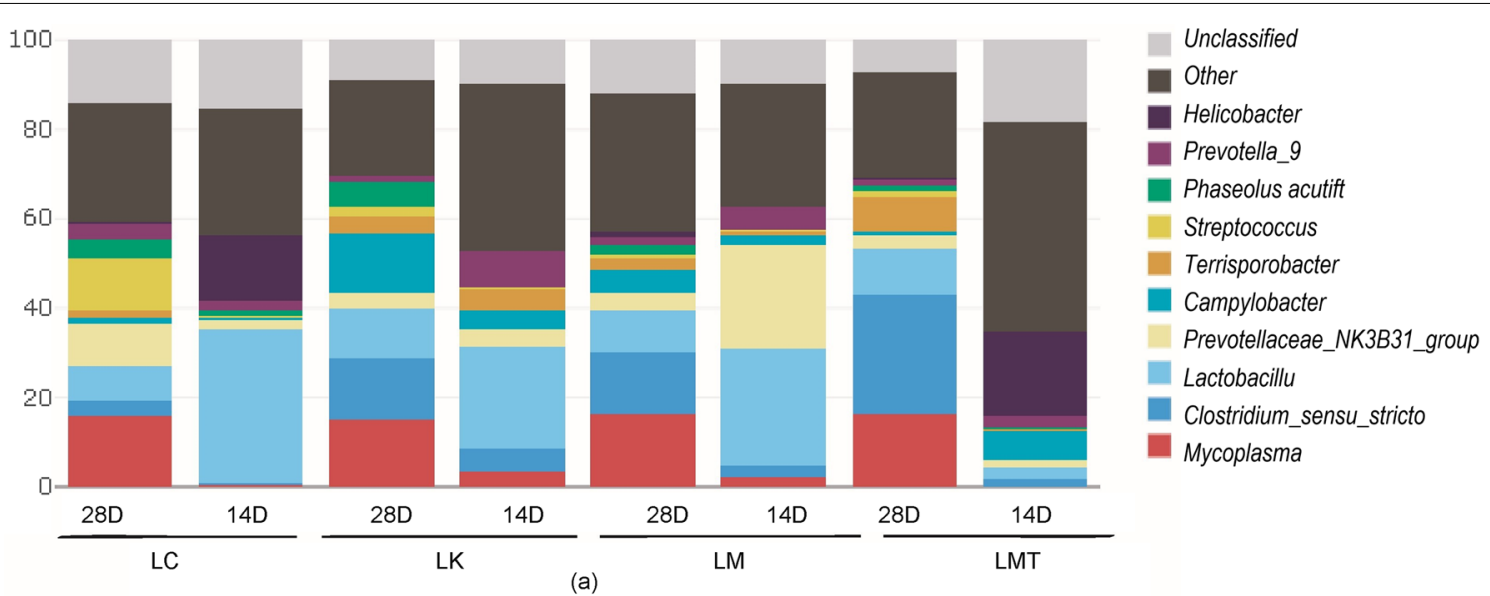

Lactobacillus

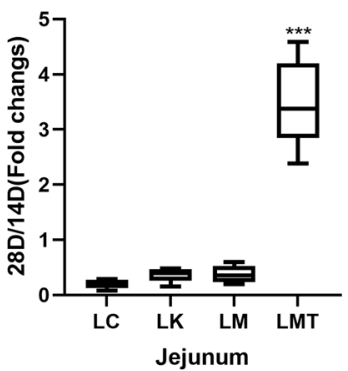

(b)
Clostridium_sensu_stricto_1

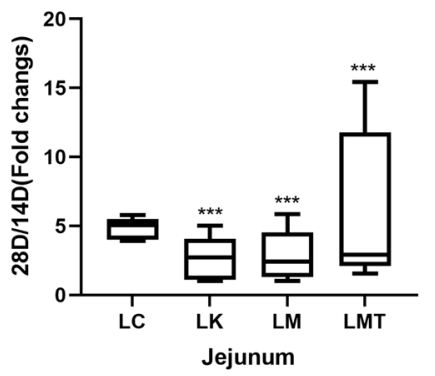

(c)

Fig. 9 Composition of the jejunum microbiota at genus and phylum levels. a The effect of the TCM formula on the composition of the jejunum microbiota at the genus level in the weaned piglets. b The fold change in the relative abundance of the genus Lactobacillus ( $28 \mathrm{~d} / 14 \mathrm{~d}$ ratio). $\mathbf{c}$ The fold change in the abundance of the genus Clostridium sensu stricto 1 ( $28 \mathrm{~d} / 14 \mathrm{~d}$ ratio) $\left({ }^{*} P<0.05 ;{ }^{*} P<0.02 ;{ }^{* *} P<0.01\right.$ )

\section{Acknowledgements}

Not applicable.

\section{Authors' contributions}

LZL, LZN, and YZY designed the study. LZL wrote the manuscript. LZL, LZN and LZ conducted the experiments. LZL edited the manuscript. All the coauthors discussed the results and reviewed the manuscript. All authors read and approved the final manuscript.

\section{Funding}

This study was funded by Fujian Science and Technology Project

\section{(2019R1021-5).}

\section{Availability of data and materials}

The datasets generated during and/or analyzed during the current study are available from the corresponding author on reasonable request.

\section{Declarations}

Ethics approval and consent to participate

All the procedures were approved by the Animal Care Review Committee (Approval No. 2020000335580).

\section{Consent for publication}

Not applicable.

\section{Competing interests}

The authors declare that they have no competing interests.

Received: 11 July 2021 Accepted: 16 November 2021

Published online: 27 November 2021

\section{References}

Bokulich NA, Subramanian S, Faith JJ, Gevers D, Gordon JI, Knight R, Mills DA, Caporaso JG (2013) Quality-filtering vastly improves diversity estimates from Illumina amplicon sequencing. Nat Methods 10(1):57-59

Burnell TW, Cromwell GL, Stahly TS (1988) Effects of dried whey and copper sulfate on the growth responses to organic acid in diets for weanling pigs. J Anim Sci 66(5):1100

Chandrakar S, Gupta AK (2015) Antibiotic potential of endophytic actinomycetes of medicinal herbs against human pathogenic bacteria. Proc Natl Acad Sci India 87(3):1-11

Chen XM, Xiao-Liang LI, Tang LP, Yin Z, Dong-You YU, Fang WH (2007) Effects of Chinese herb residues on growth performance, intestinal microflora, and immune function of weaning piglets. Acta Agric 19(1):54 
Clark GWB, Jamieson IR, Hinder RA, Polishuk PV, Demeester TR, Gupta N, Cheng SC (2010) The relationship between gastric pH and the emptying of solid, semisolid and liquid meals. Neuro-Gastroenterol Motil 5(4):273-279

Clemente JC, Ursell LK, Parfrey LW, Knight R (2012) The impact of the gut microbiota on human health: an integrative view. Cell 148(6):1258-1270

Desantis TZ, Hugenholtz P, Keller K, Brodie EL, Larsen N, Piceno YM, Phan R, Andersen GL (2006) NAST: a multiple sequence alignment server for comparative analysis of $16 \mathrm{~S}$ rRNA genes. Nucleic Acids Res 34(Web Server issue):W394-W399

Edgar RC (2013) UPARSE: highly accurate OTU sequences from microbial amplicon reads. Nat Methods 10(10):996-998

Edgar C, Jongen PJ, Sanders E, Sindic C, Goffette S, Dupuis M, Jacquerye P, Guillaume D, Reznik R, Wesnes K (2011) Cognitive performance in relapsing remitting multiple sclerosis: A longitudinal study in daily practice using a brief computerized cognitive battery. Bmc Neurol 11(1):1-11

Fan Z (2017) Coix: Chemical composition and health effects. Trends Food Sci Technol 61(Complete):160-175

Gui YF (2007) Effects of Chinese herbal formula on growth performance and serum biochemical parameters in weaned piglets. J Grad Sch Chinese Acad Sci 13:84-89

He QH, Kong XF, Hou YQ, Yin YL, Yin FG, Liu HJ, Li TJ, Huang RL, Hai Y, Gong JHBT (2007) Effects of Chinese herbal ultra-fine powder as a dietary additive on gut microbiota in early-weaned piglets. Standard Herbal Ayurvedic Formul 23:84-88

Hidaka Y, Kaneda T, Amino N, Miyai K (1992) Chinese medicine, Coix seeds increase peripheral cytotoxic T and NK cells. Biotherapy 5(3):201-203

Jian LI (2007) Effect of compound traditional chinese medicine preparations on growth performance and digestive enzyme activities of piglets. J Anhui Agric Sci 6:111-119

Kong XF, Deng ZYBT-P in FAA\& C for AP. Acanthopanax senticosus extracts as a dietary additive enhances growth performance and apparent ileal digestibilities of amino acids in weaned piglets. 2009; 157-167.

Koo HH, Chung SH (1994) Effects of Panax ginseng and Ganoderma lucidum extract on the growth of lactic acid bacteria. Korean J Food Nutr 7(1):45-50

Lai B, Lin Q, Lin B, Zhong Jl (2015) Effects of a self-made compound chinese herbal medicine additive on growth performance and occurrence of diarrhea in weaned piglets. Agric Sci Technol 23:393-398

Li H, Zhou M, Zhao A, Jia W (2010) Traditional Chinese medicine: balancing the gut ecosystem. Phyther Res Ptr 24(1):1332-1335

Li Z, Lin Z, Lu Z, Feng Z, Ying Z (2019) Coix seed improves growth performance and productivity in post-weaning pigs by reducing gut $\mathrm{pH}$ and modulating gut microbiota. AMB Express 9(1):115

Lin XL, Lin BQ, Pan SL, Lai BS, Lin QM, Lian QX, Jian-Hong LI (2013) Effect of TCM on growth and immune-response to hog cholera vaccine of weaned piglets carrying naturally acquired porcine circovirus type 2 . Fujian J Agric Sci 63(12):1393-1394

Liu S, Li F, Zhang X (2019) Structural modulation of gut microbiota reveals Coix seed contributes to weight loss in mice. Appl Microbiol Biotechnol 15:93-99

Magoä T, Salzberg SL (2011) FLASH: fast length adjustment of short reads to improve genome assemblies. Bioinformatics 27(21):2957-2963

MEler A, Wintermann M, Sander SJ, Kamphues J (2012) Effect of diet grinding and pelleting fed either dry or liquid feed on dry matter and $\mathrm{pH}$ in the stomach of pigs and the development of gastric ulcers. J Anim Sci 90 Suppl 4(13):343

Miller B, James PS, Smith MW (1986) Effect of weaning on the capacity of pig intestinal villi to digest and absorb nutrients. J Agric Sci 107:579-590

Modesto M, D'Aimmo MR, Stefanini I, Trevisi P, De FS, Casini L, Mazzoni M, Bosi P, Biavati B (2009) A novel strategy to select Bifidobacterium strains and prebiotics as natural growth promoters in newly weaned pigs. Livest Sci 122(2):248-258

Nadkarni MA, Martin FE, Jacques NA, Hunter N (2002) Determination of bacterial load by real-time PCR using a broad-range (universal) probe and primers set. Microbiology 148:257-266. https://doi.org/10.1099/00221 287-148-1-257

Nemechek JE, Tokach MD, Dritz SS, Goodband RD, Derouchey JM, Bergstrom JR et al (2013) Evaluation of antibiotics and benzoic acid on growth performance of nursery pigs. Proc 2013 Kansas Swine Day 35(2):92-98

Normile D (2003) The new face of traditional Chinese medicine. Science 299:188-190
Schnabel E, Schneider R, Schubert C (1982) pH value in the anterior tract of weanling piglets. Arch Tierernahr 32(9):631

Shao-Hua HU, Xiao XN, Xing Yl, Zhang JP (2009) Study Progress of Coix Seed Lishizhen. Med Mater Medica Res 39:94-97

Shi Q, Gao G, Xing C, Liu H, Shen P, Pan F, Gao G, Zhang Y (2015) Effects of traditional Chinese medicine on numbers of lymphocytes and goblet cells in villus epithelia of layers under heat stress. Agri Sci and Tech 09:24-37

Straw ML, Kornegay ET, Evans JL, Wood CM (1991) Effects of dietary pH and phosphorus source on performance, gastrointestinal tract digesta, and bone measurements of weanling pigs. J Anim Sci 69(11):4496-4504

Suiryanrayna MVAN, Ramana JV (2015) A review of the effects of dietary organic acids fed to swine. J Anim Sci Biotechnol 6(1):45

Wang X, University GA, Medicine COV (2010) In vitro bacteriostasis of eight Chinese herbal medicines on common pathogenic bacteria. J Gansu Agric Univ 45(2):25-29

Wang ZG, Ren J (2002) Current status and future direction of Chinese herbal medicine. Trends Pharmacol Sci 23(8):347-348

Wang X, Huang M, Yang F, Sun H, Zhou X, Guo Y, Wang X, Zhang M (2015) Rapeseed polysaccharides as prebiotics on growth and acidifying activity of probiotics in vitro. Carbohydr Polym 125(1):232-240

Wu Q, Chen H, Lv Z, Li S, Hu B, Guan Y, Xie B, Sun Z (2013) Oligomeric procyanidins of Lotus seed pod inhibits the formation of advanced glycation end-products by scavenging reactive carbonyls. Food Chem 138(2-3):1493-1502

Xi G, Liu W, Chen M, Li Q, Hao X, Wang M, Yang X, Feng Y, He H, Shi C, Li W (2019) Polysaccharide-based lotus seedpod surface-like porous microsphere with precise and controllable micromorphology for ultrarapid hemostasis. ACS Appl Mater Interfaces 11(50):46558-46571

Xu M, Dong Y, Du S, Hao YS, Wang YH, Wang FN, Yao JH (2009) Effect of corn particle size on mucosal morphology and digesta $\mathrm{pH}$ of the gastrointestinal tract in growing goats. Livest Sci 123(1):34-37

Xu CL, Zhao YF, Shang XY, Niu WN (2012) The effects of supplementing diets with Atractylodes macrocephala Koidz rhizomes on growth performance and immune function in piglets. J Anim Feed Sci 21 (2):302-312

Xu M, He D, Teng H, Chen L, Song H, Huang Q (2018) Physiological and proteomic analyses of coix seed aging during storage. Food Chem 260:82-89

Xuan W, Bai D, Zhu G, Ji Y, Jia Z, Peng Z. Isolation, identification, antibacterial effects of antibiotic drugs, and Chinese herbal extracts to the pathogenic Bacteria of Swollen Abdomen from Scophthalmus maximus in vitro. 2014; 9:123-129

Yang H, Xia X, Yin Y. Development and renewal of intestinal villi in pigs. 2013.

Yen GC, Der DuhP, Su HJ (2005a) Antioxidant properties of Lotus seed and its effect on DNA damage in human lymphocytes. Food Chem 89(3):379-385

Yen GC, Der DuhP, Su HJ (2005b) Antioxidant properties of Lotus seed and its effect on DNA damage in human lymphocytes. Food Chem 89:379-385

Yi Z, Xu L, Zeng S, Huang X, Guo Z, Zheng Y, Tian Y, Zheng B (2015) Nutritional composition, physiological functions and processing of Lotus (Nelumbo nucifera Gaertn.) seeds: a review. Phytochem Rev 14(3):321-334

Zeng H, Zheng Y, Yan L, Huang C, Shan L, Zheng B, Yi Z (2018) Effect of fractionated Lotus seed resistant starch on proliferation of Bifidobacterium longum and Lactobacillus delbrueckii subsp. bulgaricus and its structural changes following fermentation. Food Chem 04:121-134

Zhang Y, Wang Y, Zheng B, Lu X, Zhuang W (2013) The in vitro effects of retrograded starch (resistant starch type 3) from Lotus seed starch on the proliferation of Bifidobacterium adolescentis. Food Funct 4(11):1609-1616

Zhang Y, Zeng H, Wang Y, Zeng S, Zheng B (2014) Structural characteristics and crystalline properties of Lotus seed resistant starch and its prebiotic effects. Food Chem 155(19):311-318

Zhao X, Lin L, Luo Q, Ye M, Luo G, Kuang Z (2015) Effects of mulberry (Morus alba $\mathrm{L}$.) leaf polysaccharides on growth performance, diarrhea, blood parameters, and gut microbiota of early-weanling pigs. Livest Sci 177:88-94

Zu XP, Lin Z, Xie HS, Yang N, Liu XR, Zhang WD (2016) Interaction of effective ingredients from traditional Chinese medicines with intestinal microbiota. China J Chinese Mater Medica 41(10):1766-1772

\section{Publisher's Note}

Springer Nature remains neutral with regard to jurisdictional claims in published maps and institutional affiliations. 